\title{
Fisetin inhibits proliferation of pancreatic adenocarcinoma by inducing DNA damage via RFXAP/KDM4A-dependent histone H3K36 demethylation
}

\author{
Guoping Ding ${ }^{1}$, Xiaodong $\mathrm{Xu}^{1}$, Dan $\mathrm{Li}^{1,2}$, Yuhao Chen ${ }^{1,3}$, Weimin Wang ${ }^{1,4}$, Dongnan Ping ${ }^{1}$, Shengnan Jia ${ }^{1}$ and \\ Liping $\mathrm{CaO}^{1,15}$
}

\begin{abstract}
Pancreatic adenocarcinoma (PDAC) is an extremely malignant tumor that is associated with low survival rates. Fisetin is a natural flavonoid that shows diverse antitumor effects, including DNA damage, in various cancers. Increasing studies have demonstrated that epigenetic modifications play critical roles in DNA-damage response. However, the epigenetic regulation mechanism of fisetin in cancers is hardly studied. RFXAP is a critical transcription factor for MHC II molecules, however, its transcriptional role in PDAC is poorly understood. The anti-PDAC effect of fisetin was measured by CCK-8, flow cytometry, xenograft tumor nude mice model. DNA-damage levels were examined by immunofluorescence. Bioinformatics analysis was used to examine the expression of RFXAP and other genes involved in DNA-damage response. ChIP sequencing was used to explore the transcriptional role of RFXAP. The expression of target gene KDM4A was measured by qRT-PCR and western blots. KDM4A promoter activity was analyzed using dual-luciferase reporter assay. RFXAP overexpressing or silencing of PDAC cells was used to explore the effect of RFXAP in DNA damage induced by fisetin. We found that fisetin inhibited cell proliferation and induced DNA damage and S-phase arrest in PDAC. Expression of RFXAP and other DNA-damage response genes were upregulated by fisetin. We revealed that RFXAP expression was relatively low in PDAC and correlated with tumor stage and poor prognosis. Then we explored the transcriptional role of RFXAP and found that RFXAP targeted KDM4A, a special demethylase specific for tri- and dimethylated histone H3K36. We found that overexpression of RFXAP upregulated KDM4A and attenuated methylation of H3K36, thereby impairing DNA repair and enhancing the DNA damage induced by fisetin, while RFXAP silencing showed the opposite effect. We also found the function of fisetin in enhancing the effect of chemotherapy on pancreatic cancer cells. Our findings revealed that fisetin induced DNA damage via RFXAP/KDM4A-dependent histone H3K36 demethylation, thus causing inhibition of proliferation in PDAC.
\end{abstract}

Correspondence: Shengnan Jia (jiashengnan8180@zju.edu.cn) or Liping Cao (caolipingzju@zju.edu.cn)

'Department of General Surgery, Sir Run Run Shaw Hospital, School of Medicine, Zhejiang University, Hangzhou 310000, China

${ }^{2}$ Department of General Surgery, School of Medicine, Affiliated Hospital of Hangzhou Normal University, Hangzhou 310000, China

Full list of author information is available at the end of the article

These authors contributed equally: Guoping Ding, Xiaodong Xu

Edited by A. Stephanou

\section{Introduction}

Pancreatic adenocarcinoma (PDAC) is an extremely malignant tumor characterized by low rates of early diagnosis and radical resection ${ }^{1}$. Patients with PDAC frequently develop resistance to chemotherapy and radiotherapy, and the prognosis of these patients is poor, with 5-year survival rates of $3-6 \%^{2}$. It is the fourth leading cause of cancer-related death in the United States and the fifth in China ${ }^{3,4}$.

\section{(c) The Author(s) 2020}

(c) (i) Open Access This article is licensed under a Creative Commons Attribution 4.0 International License, which permits use, sharing, adaptation, distribution and reproduction cc. in any medium or format, as long as you give appropriate credit to the original author(s) and the source, provide a link to the Creative Commons license, and indicate if changes were made. The images or other third party material in this article are included in the article's Creative Commons license, unless indicated otherwise in a credit line to the material. If material is not included in the article's Creative Commons license and your intended use is not permitted by statutory regulation or exceeds the permitted use, you will need to obtain permission directly from the copyright holder. To view a copy of this license, visit http://creativecommons.org/licenses/by/4.0/. 
Because of uncontrolled proliferation, cancer cells are more likely to incur DNA damage including doublestrand breaks (DSBs) and single-strand breaks ${ }^{5}$. Excessive DSBs beyond the capacity for cellular repair, induced by radiation or chemotherapeutic drugs such as cisplatin and etoposide, cause death of replicating cells; however, cancer cells with elevated DNA repair mechanisms may have acquired and intrinsic drug resistance, and thus poor chemotherapy outcomes ${ }^{6,7}$. Recent studies have demonstrated that inhibition of DNA repair pathways in cancer cells can significantly enhance the effects of chemotherapy and radiotherapy, ${ }^{8,9}$.

H3K36 methylation plays a conserved role in regulating DNA repair in cancer. Recent studies have demonstrated that SETD2-dependent H3K36me3 is critical for homologous recombination (HR) repair ${ }^{10,11}$. Interestingly, the trimethylation of H3K36 is not induced by DNA damage but is pre-existing ${ }^{12}$. KDM4A is a demethylase specific for tri- and dimethylated histone H3K36 and H3K9, which causes demethylation of histone $\mathrm{H} 3 \mathrm{~K} 36$ and thereby impairs $\mathrm{HR}^{10}$.

Regulatory factor $\mathrm{X}$-associated protein (RFXAP) is a critical transcription factor for MHC II molecules ${ }^{13,14}$. We previously found that miR-212-3p transfers from pancreatic cancer cells to dendritic cells via exosomes, inhibiting expression of RFXAP and major histocompatibility complex (MHC) class II molecules and leading to immune tolerance of dendritic cells ${ }^{15}$ miR-212-3p is upregulated in PDAC and is correlated with TNM stage and poor prognosis ${ }^{16-18}$. Thus, we hypothesized that miR212-3p-induced RFXAP deficiency may occur in PDAC. Deficiency of RFXAP can lead to inactivation of CD4+ T lymphocytes, which is a rare and severe immunodeficiency disease termed bare lymphocyte syndrome ${ }^{19}$. However, the role of RFXAP deficiency in malignant tumors has not been documented.

Fisetin, as a natural flavonoid, is mainly present in various vegetables and fruits such as apples, cucumbers, onions, and strawberries ${ }^{20}$. Fisetin is known to possess diverse pharmacological activities, such as antioxidant ${ }^{21}$, anti-inflammatory ${ }^{22}$, and antitumor effects in various cell types $^{23-25}$. From these reports, it appears that the antitumor mechanism of fisetin may be cancer-cell-specific. Recent studies reported that fisetin can induce DNA damage and apoptosis in gastric and hepatic cancer and enhance the DNA-damage-induced toxicity of etoposide $^{25-27}$. However, there have been no studies that focused on the DNA damage induced by fisetin in PDAC.

In this study, we found that the expression of RFXAP was relatively low in pancreatic cancer, and its downregulation was correlated with tumor stage and poor prognosis. RFXAP was shown to bind to the promoter region of the KDM4A gene and induce its expression, affecting DNA repair. We have demonstrated that fisetin can induce DNA-damage cytotoxicity in PDAC through regulation of RFXAP/KDM4A-dependent demethylation of H3K36me3, thereby impairing DNA HR repair. The present results suggest that fisetin-induced RFXAP and KDM4A play an important role in antitumor processes by causing DNA damage and DNA repair dysfunction.

\section{Materials and methods \\ Reagents and antibodies}

Primary antibodies used in this study were as follows: polyclonal anti-a-tubulin (Sigma, catalog no.T6199), antiRFXAP (Abcam, ab9258; ab87251), anti-KDM4A (Abcam, ab191433), anti-H3 (Abcam, ab201456), anti-H3K36me3 (Abcam, ab9050), anti- $\gamma-\mathrm{H} 2 \mathrm{AX}($ Abcam, ab26350; Cell Signaling Technology, catalog no. \#9718S).

\section{Cell culture}

The human pancreatic cancer-cell lines BxPC-3, MiaPACA-2, PANC-1, and HPC-Y5 and the normal pancreatic ductal epithelial cell line HPDE6-C7 were obtained from the Chinese Academy of Sciences (Shanghai, China). BxPC-3 cells were cultured in RPMI 1640 medium (Hyclone) containing 10\% fetal bovine serum (FBS, Gibco, New York, USA). PANC-1, HPC-Y5, MiaPACA-2, and HPDE6-C7 cells were cultured in DMEM supplemented with 10\% FBS.

\section{Patients and tissue specimens}

Forty-six PDAC patients who underwent surgery between 2012 and 2016 were selected from Sir Run Run Shaw Hospital, affiliated with the Zhejiang University of Medicine, China. All tissue specimens were diagnosed by pathological examination after surgery. The mean age of the patients was 59.0 years (range, 35-82). The male to female ratio was 22:24. The research protocol was reviewed and approved by the Research Ethics Committee of Sir Run Run Shaw Hospital, School of Medicine, Zhejiang University. All participants or their guardians gave written consent for the use of tissue samples and medical information to be used for scientific research.

\section{Clinicopathological parameters}

Clinicopathological factors including age, gender, tumor size, differentiation, histological stage, lymph node metastasis, and distant metastasis were retrospectively analyzed. Survival data were reviewed in all cases. The histological stage of PDAC patients was assessed on the basis of the newest TNM staging system described by the American Joint Committee on Cancer (AJCC) in 2017.

\section{Immunohistochemistry}

Immunohistochemical staining was performed using the standard streptavidin-biotin-peroxidase complex method. Briefly, paraffin-embedded tissue sections were dewaxed and rehydrated through an alcohol series, and endogenous peroxidase activities were blocked. Slides 
were incubated overnight at $4{ }^{\circ} \mathrm{C}$ with monoclonal antiRFXAP (Abcam, ab87251). Samples incubated with PBS instead of primary antibody were used as the negative control. Proteins were detected using secondary antibodies and counterstained in Gill's hematoxylin, then dehydrated in an ascending methanol series prior to clearing in xylene and mounting under a coverslip. The sections were observed under an Olympus CX31 microscope (Olympus, Tokyo, Japan).

Cells with the cytoplasm stained yellow to brown were scored as RFXAP immunopositive. RFXAP expression was classified semiquantitatively according to the following criteria:,$-<1 \%$ of neoplastic cells discretely expressing RFXAP;,$+ \geq 1$ of morphologically unequivocal neoplastic cells discretely expressing RFXAP.

\section{Transfection and siRNA}

For transduction, cells were transfected with the appropriate target plasmid and packaging constructs overnight, and the viral supernatant was collected $48 \mathrm{~h}$ later. PANC-1 and BxPC-3 cells were transduced in the presence of $5-\mu \mathrm{g} / \mathrm{ml}$ polybrene and selected for 3-7 days depending on the level of EGFP expression. RFXAP siRNAs (Ribobio, China) were used to knock down RFXAP gene expression. The RFXAP and control siRNA (100 pM) were transfected into PANC-1 cells using siRNA transfection reagent (Santa Cruz Biotechnology) $24 \mathrm{~h}$ before fisetin treatment. The cells were collected after fisetin treatment for $48 \mathrm{~h}$. The Ubi-MCS-LucIRES-Puromycin vector was used to construct PANC-1luciferase cells. Cells were transfected using Lipofectamine 3000 (Invitrogen, USA) and selected for puromycin resistance. KDM4A-knockout PANC-1 cells were constructed using the CRISPR/Cas9 gene-editing system. The target sequences of gRNAs were designed using the online tool (https://www.benchling.com/crispr/), which listed as follows: KDM4A-cas9 (5'-AAGGTCATTATCCTAGCAC T-3'). Plasmid Mini-PREPS Kit (Sangon Biotech, catlogB 110091-0100) was used for plasmid extraction. CRISPR plasmids were transfected using Lipofectamine 3000 (Invitrogen, USA) and selected for puromycin resistance.

\section{Dual-luciferase reporter assay}

PANC-1 and BxPC-3 cells pre-transfected with pcDNA3.1 (-) (Invitrogen, USA) or pcDNA-RFXAP (RFXAP overexpression) vectors were co-transfected with the pGL4.23KDM4A promoter-luc reporter vector. pRL-TK vector (Promega, Madison, WI, USA) was used as an internal control. Cell lysates were harvested for the dual-luciferase assay, which was performed according to the manufacturer's instructions (Promega, Madison, WI, USA).

\section{Animals}

Nude mice were obtained from SLAC Laboratory and bred in the animal center of Zhejiang University under specific pathogen-free conditions. Orthotopic PDAC model was established with human pancreatic cancer PANC-1-luciferase cells. Tumor growth and the condition of mice were monitored every other day. Animal experiments were approved and performed in accordance with the institutional guidelines for animal care of the animal ethics committee of Zhejiang University. For the detection of bioluminescence, mice were injected intraperitoneally with $200 \mu \mathrm{L}(15 \mathrm{mg} / \mathrm{mL})$ of D-luciferin. Tumors were imaged after $15 \mathrm{~min}$ of biodistribution, with an IVIS Lumina Series III (Perkin Elmer, USA).

\section{Gene co-expression analysis}

RNA-Seq data of 182 pancreatic cancer samples were collected from TCGA (http://cancergenome.nih.gov). The RNA-Seq data were transformed into $\log 2$ scale. Based on the correlation analyses, genes coexpressed with RFXAP were identified according to a Pearson's correlation coefficient $>0.5$.

\section{ChIP-sequencing data analysis}

FastQC was used as a stand-alone interactive application for the immediate analysis of FastQ files to determine whether the ChIP-seq data had any problems before the mapping analysis. The sequenced DNA tags (49 bp) were all sequence reads generated from Illumina HiSeq 2500 and were aligned against the human reference sequence (hg19, June 2011) using the Bowtie 2 tool, allowing one mismatch in the default seed length of 49 bases. The sequence reads mapped to the genome were subjected to peak calling to detect regions with significant enrichment of ChIP signals with respect to the background by using MACS2. Motifs were searched both in the full list of peaks and in subsets of peaks divided by enrichment, and location, and sequences 2-kb upstream of the transcription start site were used as background. The DAVID software was used for Gene Ontology and Pathway analyses.

\section{Apoptosis and cell-cycle analysis}

The levels of cell apoptosis were tested using the BD FITC Annexin-V Apoptosis Detection Kit I (556547). Cells were analyzed by flow cytometry after $1 \mathrm{~h}$. Cell cycle was detected by the Cell-Cycle Staining Kit (MultiSciences, China, 70-CCS012).

\section{Immunofluorescence via confocal microscopy}

PDAC cells were fixed with $4 \%$ paraformaldehyde at $4{ }^{\circ} \mathrm{C}$ overnight and then washed with $\mathrm{PBS}$ in 5 min for three times. Fixed cells were blocked in antibody dilution buffer (PBS containing $0.25 \%$ Triton X-100 and 1\% bovine serum albumin), and all subsequent staining was performed in the same buffer. The cells were labeled with primary antibody for $2 \mathrm{~h}$ and subsequently with Alexa Fluor 488-conjugated donkey anti-rabbit secondary 
antibody (Invitrogen Life Technologies) for $1.5 \mathrm{~h}$. The nucleus was stained by incubation with DAPI for $15 \mathrm{~min}$ at room temperature. Cells were scanned using a ZEISS LSM 880 confocal microscope (Zeiss, Jena, TH, GER).

\section{Statistical analysis}

Data were analyzed using SPSS version 20.0. The Kaplan-Meier method was used to analyze patient survival, and the differences in survival were evaluated using the log-rank test. Multivariate analysis was performed using the Cox proportional hazards model to confirm factors independently associated with survival. The paired-samples $t$-test was used to compare the intensity ratio between HCC tissues and matching noncancerous liver tissues. All $P$ values were based on two-sided statistical analyses, and $P<0.05$ was considered statistically significant.

\section{Results}

Fisetin inhibited the viability of human pancreatic cancer cells in vitro and in vivo

We measured the effect of fisetin on the proliferation and growth of four pancreatic cancer-cell lines (PANC-1, BxPC-3, MiaPACA-2, HPC-Y5) using a Cell Counting Kit-8 assay. Cancer cells were cultured with increasing concentrations of fisetin $(0,25,50,100,200$, and $400 \mu \mathrm{M})$, and the results showed that fisetin reduced pancreatic cancer-cell viability in a dose-dependent manner (Fig. 1a, b and Supplementary Fig. S1A, B). Interestingly, we found that low concentrations of fisetin $(25,50 \mu \mathrm{M})$ did not inhibit the viability of HPC-Y5 and PANC- 1 cells efficiently, while BxPC-3 and MiaPACA-2 were sensitive to low concentration of fisetin. Thus, we hypothesized that the differences in sensitivity to fisetin may be cellspecific.

Although studies on the antitumor effect of fisetin have increased over recent years, there have been few studies about its in vivo antitumor activity, especially in PDAC. To determine the in vivo efficacy of fisetin, we selected PANC-1 cells with low sensitivity to fisetin in vitro and used a nude mouse xenograft model of luciferaseexpressing PANC-1 tumor cells, which emit bioluminescence after intraperitoneal luciferin injection. This allowed the noninvasive detection of tumor size and position in living mice. Notably, fisetin significantly reduced tumor size in mice (Fig. 1c). The tumor volumes $30 \mathrm{~d}$ after treatment were $1631.0 \pm 243.3 \mathrm{~mm}^{3}$ and $483.7 \pm$ $100.7 \mathrm{~mm}^{3}$ (mean \pm standard deviation) in the control and fisetin treatment groups, respectively (** $P<0.01$; Fig. $1 d$ ). Although compared with BxPC-3 and MiaPACA-2, PANC-1 cells were not very sensitive to fisetin in vitro, the antitumor effects of fisetin were still significant. Overall, these results demonstrated that fisetin can significantly inhibit PDAC growth and invasion in vivo.
To investigate whether fisetin-induced PDAC cell death occurred via the apoptosis, we selected PANC-1 and BxPC-3 cells to assess phosphatidylserine translocation using Annexin- $\mathrm{V}$ and propidium iodide double-staining. Apoptosis analysis by flow cytometry showed a increased number of apoptotic cells in the fisetin treatment group (Fig. 1e, f). The proportion of cells of early apoptotic cells (Annexin-V positive) were $14.98 \% \pm 0.67 \%\left({ }^{* * * *} P<0.001\right)$ and $13.69 \% \pm 0.89 \%\left({ }^{* * * *} P<0.001\right)$ after $48 \mathrm{~h}$ of treatment with $100-\mu \mathrm{M}$ fisetin for PANC-1 cells and $50-\mu \mathrm{M}$ fisetin for BxPC-3 cells, respectively. These results demonstrated the ability of fisetin to induce apoptosis in PDAC cells.

\section{Fisetin induced $\mathrm{S}$ phase and DNA damage in pancreatic cancer cells}

We found that fisetin also induced S-phase arrest in both PANC-1 and BxPC-3 cell lines. The results showed that time-dependent downregulation of G1-phase and upregulation of S-phase cells in both PANC-1 and BxPC3 cells with treatment (Fig. 2a, b).

It is well known that many chemotherapeutic drugs such as gemcitabine and etoposide cause S-phase arrest and DNA damage, thereby inducing apoptosis. Thus, we hypothesized that fisetin might induce DNA damage in PDAC. To examine DNA-damage levels, we used immunofluorescence to detect gamma-H2AX foci, which is a DNA-damage marker (Fig. 2c). The results showed that gamma-H2AX foci levels were significantly increased in PDAC cells with fisetin treatment (Fig. 2d). To figure out the mechanism of DNA damage induced by fisetin, we analyzed our previous RNA-sequencing results, GSE117189. The analysis identified 2672 differentially regulated genes $(P<0.05, q<0.05$, fold-change $\geq 2)$, of which 1136 and 1536 were up- and downregulated, respectively (Fig. 2e). We found that the mRNA levels of RFXAP and diverse DNA repair genes such as RAD50, $B R C A 2$, RPA2, and POLD3 were upregulated. We further confirmed the results of RNA sequencing by quantitative RT-PCR (qRT-PCR) (Fig. 2f).

\section{Expression of RFXAP in pancreatic cancer}

A search of the Gene Expression Omnibus database (GSE15471) showed that the relative expression of RFXAP in PDAC was significantly lower than that in normal pancreatic tissues ( 4.29 vs. 4.48$)(P<0.05)$ (Fig. 3a). Based on Gene Expression Profiling Interactive Analysis

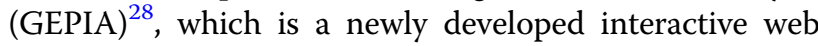
server for analyzing the RNA-sequencing data of tumors and normal samples from The Cancer Genome Atlas (TCGA) and The Genotype-Tissue Expression databases based on a standard processing pipeline, we found that RFXAP expression was significantly reduced in high-grade (stage III and IV) tumors compared with low-grade (stage I and II) tumors, and the level of RFXAP was positively 
A

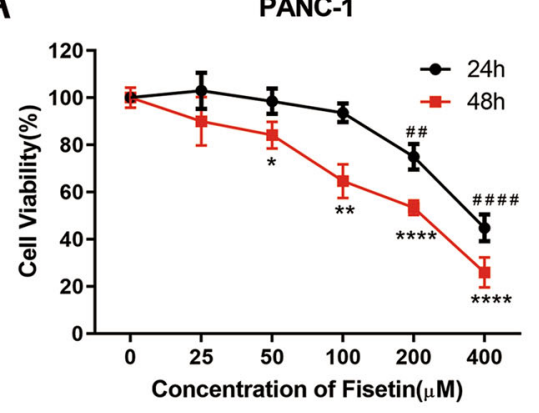

C

Control

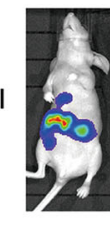

Fisetin

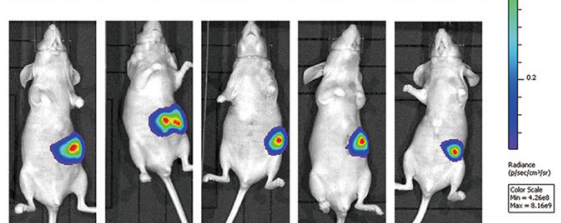

E
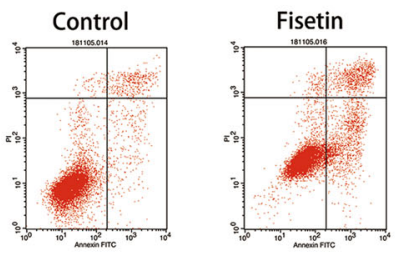

PANC-1

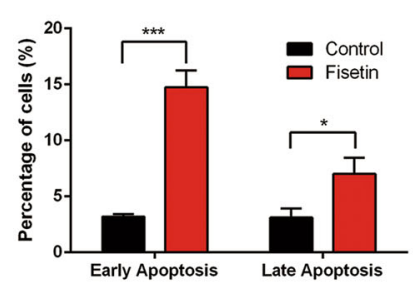

B

BxPC-3

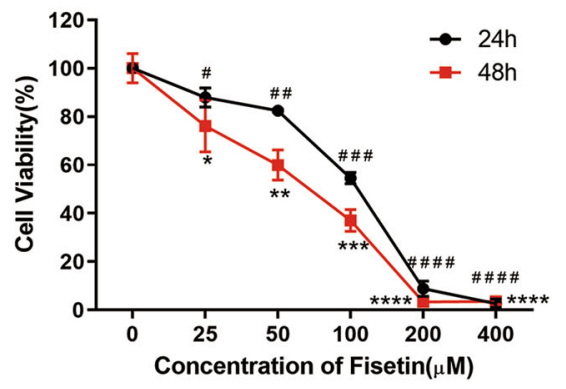

D

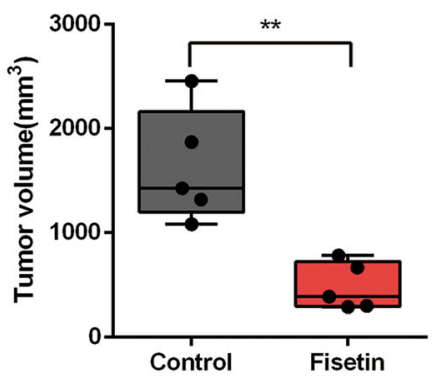

F
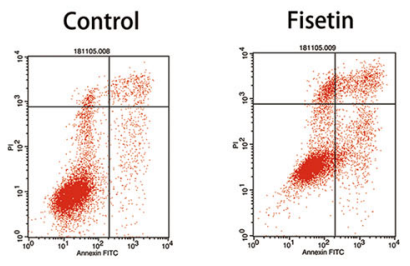

BxPC-3

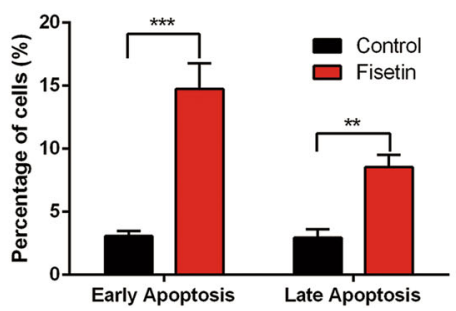

Fig. 1 Effect of fisetin on viability of PDAC in vitro and in vivo. a, b CCK-8 asssy of PANC-1 and BxPC-3 cells. Cells were treated with fisetin $(0-400 \mu \mathrm{M})$ for $24,48 \mathrm{~h}$, respectively. The absorbance was measured at $450 \mathrm{~nm}$. Data are presented as mean $\pm \mathrm{SD}(n=3) ;{ }^{*}$ or \#P<0.05, ${ }^{* *}$ or $\# \# P<0.01$, ${ }^{* *}$ or \#\#\#P<0.001, ${ }^{* * * *}$ or \#\#\#\#P<0.0001. c Orthotopic PDAC model was established with human pancreatic cancer PANC-1-luciferase cells. Control group mice were treated with DMSO via intraperitoneal injection, and treatment group mice were treated with Fisetin (160-mg/kg body weight i.p.) every other day. After 30 days treatment, mice imaged for bioluminescence. Data were expressed as average radiance (photons/s/ $\mathrm{cm}^{2} / \mathrm{sr}$ ). $\mathbf{d}$ The differences in tumor volume between control and fisetin treatment groups at 28 days after treatment. Results are mean \pm SD $(n=5)$, ${ }^{*} P<0.01$. e, f Apoptosis of cells with treatment. PANC-1 and BxPC-3 cells were treated with fisetin $(100,50 \mu \mathrm{M})$ for $48 \mathrm{~h}$, respectively, followed by Annexin-V/PI staining and flow cytometry analysis. Data are presented as mean $\pm \mathrm{SD}(n=3) ;{ }^{*} P<0.05,{ }^{* *} P<0.01,{ }^{* * *} P<0.001$.

correlated with overall survival and disease-free survival $(P<0.05)$ (Fig. 3b, c and Supplementary Fig. S1C).

We used qRT-PCR to determine the mRNA levels of RFXAP in four pancreatic cancer-cell lines (BxPC-3, MiaPACA-2, HPC-Y5, and PANC-1) and one normal pancreatic ductal epithelial cell line (HPDE6-C7). RFXAP expression was significantly lower in the pancreatic cancer-cell lines than in HPDE6-C7 cells (Fig. 3d, e).

A total of 46 pancreatic cancer tissue specimens collected in our hospital were compared with 20 paracancerous tissue specimens as the control group. Immunohistochemistry was used to detect the expression 


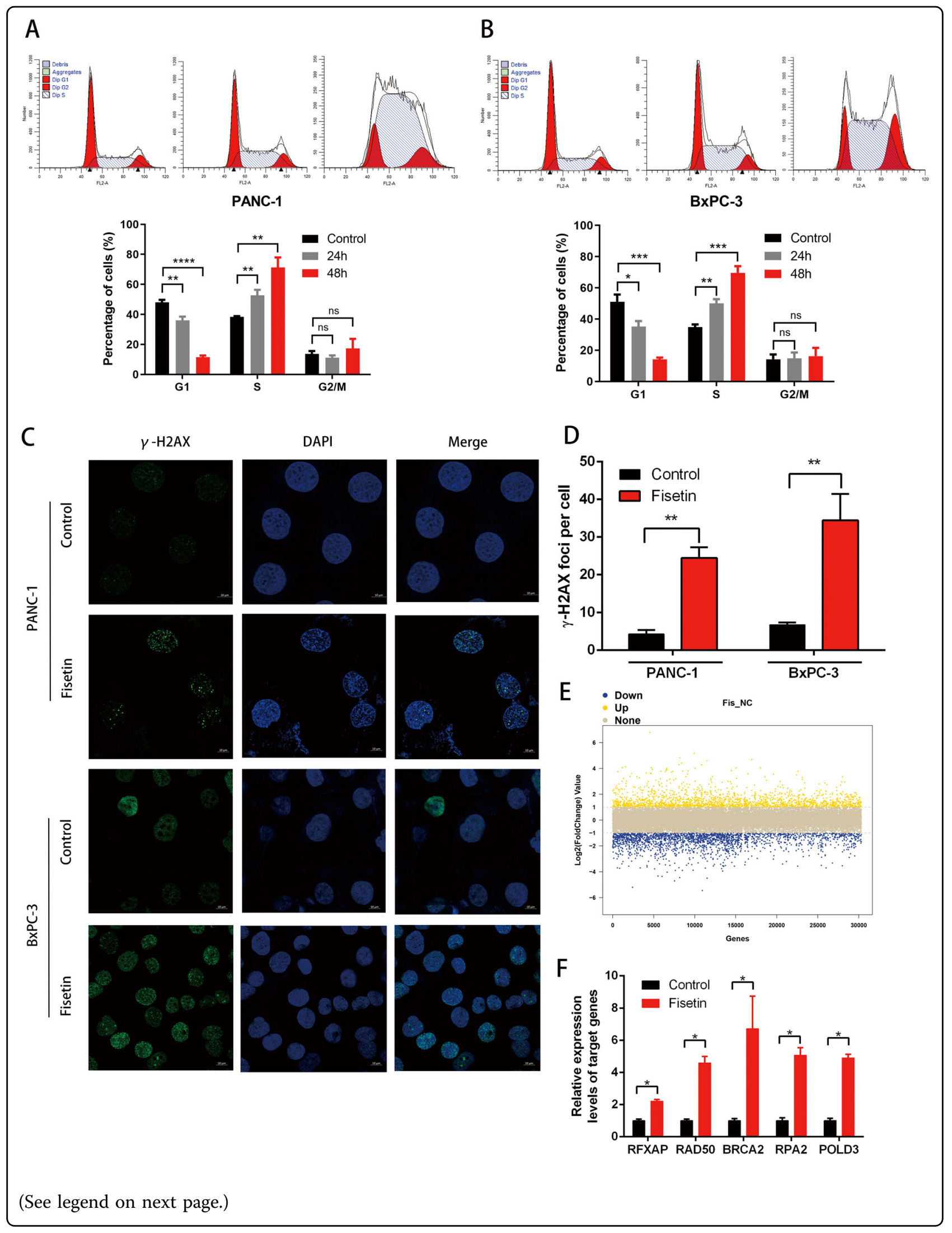


(see figure on previous page)

Fig. 2 Fisetin induces S-phase arrest and DNA damage of PDAC. $\mathbf{a}, \mathbf{b}$ Cell cycle of cells with treatment PANC-1 and BxPC-3 cells were treated with fisetin $(100,50 \mu \mathrm{M})$ for $48 \mathrm{~h}$, respectively, followed by PI staining and flow cytometry analysis. Data are presented as mean $\pm \mathrm{SD}(n=3) ;{ }^{*} P<0.05$, ${ }^{* *} P<0.01,{ }^{* * *} P<0.001,{ }^{* * * *} P<0.0001$, \#ns no significance. c Analyses of DNA damage by observation of the immunofluorescence of $\gamma$-H2AX (green) in PDAC cells. PANC-1 and BXPC-3 cells were treated with fisetin $(100,50 \mu \mathrm{M})$ for $48 \mathrm{~h}$, respectively. The nucleus were counterstained with DAPI (blue). Scale bars $10 \mu \mathrm{m}$. $\mathbf{d}$ Comparative changes in the $\mathrm{\gamma}-\mathrm{H} 2 \mathrm{AX}$ spots number in control and treatment groups. The spots were counted by manual scoring. Results are mean $\pm S D, * * P<0.01$. e RNA-sequencing analysis identified 2672 differentially regulated genes $(P<0.05, q<0.05$, fold-change $\geq 2)$, of which 1136 and 1536 were up- and downregulated, respectively. \#Fis fisetin treatment, NC negative control. Yellow and blue indicate high and low expression, respectively. $\mathbf{f}$ qRT-PCR detection of targeted genes in the RNA sequencing of PANC-1 cells. The results are presented as the mean \pm SD $(n=3), * P<0.05$.

of RFXAP in tissue samples, which showed that the RFXAP-positivity rate was significantly lower in pancreatic cancer samples than in the control group $[37.0 \%$ (17/46) vs. $65.0 \%(13 / 20) ; P<0.05$ ] (Fig. 3f). Analysis of clinicopathological data showed that negative expression of RFXAP was significantly correlated with high TNM stage, $\mathrm{T}$ stage, and poor prognosis (Table 1 and Fig. $3 \mathrm{~g}$ ).

\section{RFXAP functions in DNA damage induced by fisetin}

To investigate the molecular mechanisms underlying the role of RFXAP in PDAC, genes coexpressed with RFXAP were identified in TCGA pancreatic cancer database. Twenty-three genes were significantly coexpressed with RFXAP $(R>0.5)$, and 778 genes were coexpressed with an $R$ value $>0.3$ (Supplementary Table 1 ). The heat map for coexpressed genes is shown in Fig. 4a. Gene ontology and KEGG were used to predict signaling pathways associated with RFXAP (Fig. 4b, c and Supplementary Tables 2 and 3), and showed that RFXAP expression was correlated with cell-cycle regulation and the DNA-damage response. RFXAP was positively correlated with $R A D 50, B R C A 2, R P A 2, P O L D 3$, and other genes (Supplementary Fig. S1D). These genes play important roles in DNA-damage repair, whereas dysregulation of their expression often results in pancreatic cancer gene mutations, chemoradiotherapy resistance, and tumor progression ${ }^{29-41}$. To further examine the role of RFXAP in PDAC cells with treatment of fisetin, ChIP sequencing was performed in RFXAP-overexpressing PANC-1 (RFXAP-PANC-1) cells to explore the function of RFXAP as a transcription factor. The results showed that RFXAP interacted with the promoters of 65 genes (Table 2 and Supplementary Table 4). We further analyzed the RNA-sequencing results and found that there were 4361 differentially regulated genes $(P<0.05, q<0.05$, fold-change $>1.5$ ). Then we analyzed the overlapping genes of RFXAP co-expression, ChIP sequencing, and fisetin RNA sequencing by Venn diagrams (Fig. 4d). Based on NetworkAnalyst (http://www.networkanalyst.ca/) ${ }^{42}$, we analyzed the protein-protein interaction of the overlapping genes of RFXAP co-expression and fisetin RNAsequencing results and found that apoptosis and cell-cycle pathways were enriched (Fig. 4e and Supplementary
Table 5). We focused on KDM4A for the analysis of overlapping genes of ChIP sequencing and RNA sequencing. Recent studies showed that KDM4A can impair DNA HR repair through regulation of histone H3K36 demethylation ${ }^{10}$. Based on the GEPIA analysis, we found that RFXAP was positively correlated with $K D M 4 A$ after normalization with $G A P D H$, and patients with high $K D M 4 A$ expression showed better prognoses (Fig. 5a and Supplementary Fig. S1E, F). Thus, we hypothesized that RFXAP might be involved in fisetin-induced DNA damage in PDAC. qRT-PCR analysis further verified that $K D M 4 A$ was upregulated in PDAC treated with fisetin (Fig. 5b). Dual-luciferase reporter assays further identified the transcriptional regulation of $K D M 4 A$ by RFXAP in both PANC-1 and BxPC-3 cells (Fig. 5c, d). We also detected the protein level of KDM4A and histone H3K36me3, which can be attenuated by KDM4A, in PDAC cells with fisetin treatment. The results showed that fisetin reduced the expression of H3K36me3 (Fig. 5e).

\section{RFXAP regulates DNA damage via KDM4A-dependent H3K36 demethylation}

To further confirm whether the DNA damage observed following fisetin treatment was associated with RFXAP, we overexpressed RFXAP by pcDNA-RFXAP plasmids. qRT-PCR and western blot analysis showed the efficiency of RFXAP overexpression (Fig. 6a, b). Then we found that RFXAP overexpression enhanced the levels of KDM4A and attenuated the expression of H3K36me3 (Fig. 6c, d). Moreover, immunofluorescence showed that overexpression of RFXAP enhanced the gamma-H2AX foci levels after fisetin treatment (Fig. 6e, f), and CCK-8 assay showed that overexpression of RFXAP strengthened the antitumor effect of fisetin (Fig. 6g). Then we silenced RFXAP expression by RNAi; RFXAP expression was detected by qRT-PCR and western blot analysis. The results revealed that expression of RFXAP was reduced by silencing (Fig. 7a, b). Then we picked most-efficient RFXAP silencing siRNA (si3) to further test the effects of RFXAP silencing by western blot analysis. The results indicated that RFXAP silencing attenuated the expression of KDM4A and enhanced the levels of H3K36me3 (Fig. 7c). Immunofluorescence revealed that RFXAP 


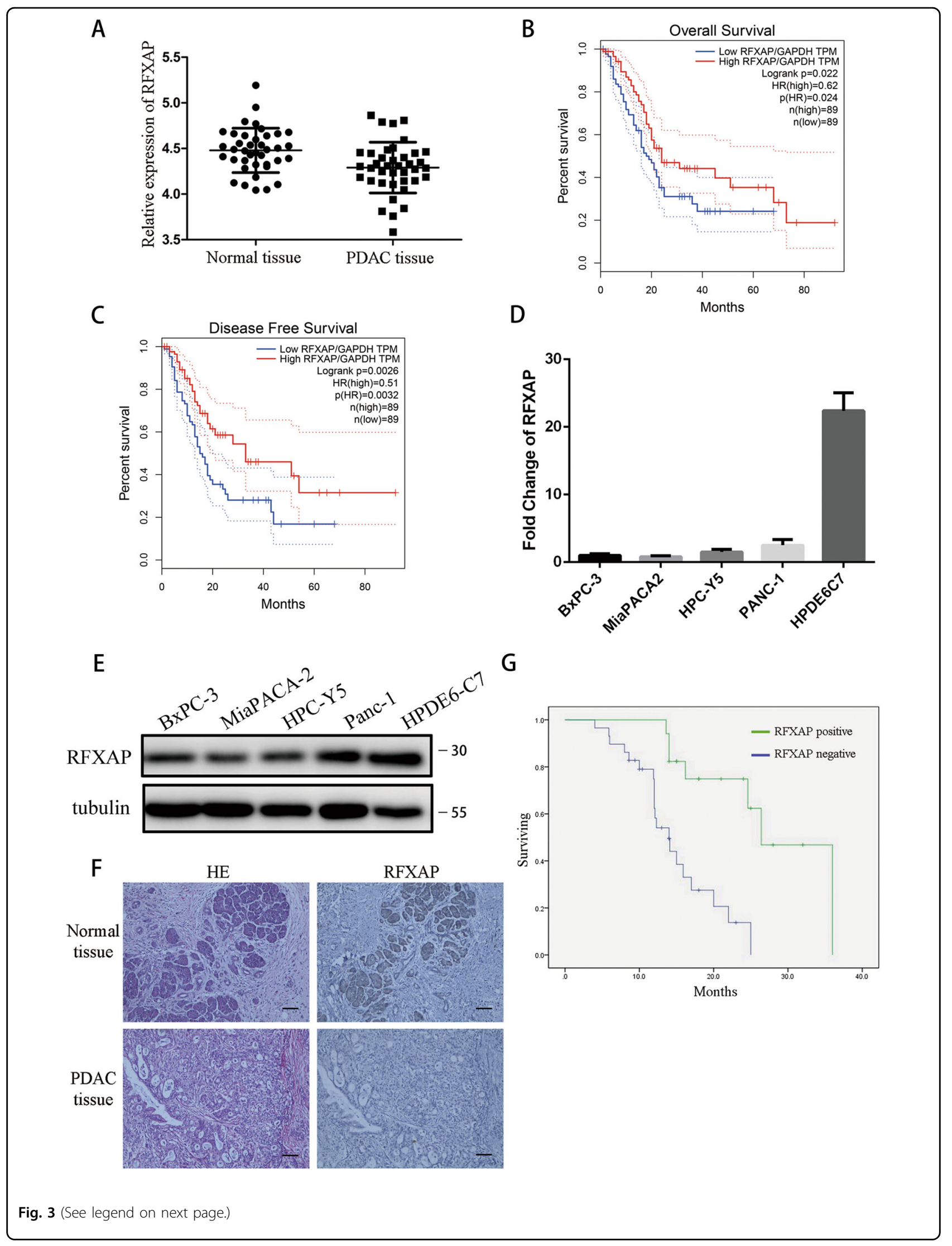


(see figure on previous page)

Fig. 3 Expression of RFXAP in pancreatic cancer and its association with prognosis. a The Gene Expression Omnibus database (GSE15471) showed that the expression of RFXAP is lower in pancreatic cancer than in normal tissues. $\mathbf{b}, \mathbf{c}$ The overall survival and disease-free survival analysis of patients with PDAC from GEPIA database. Patients with high levels of RFXAP have a better prognosis. Data are presented as mean \pm SD $(n=89)$. d GRT-PCR showed that the mRNA expression of RFXAP was lower in pancreatic cancer-cell lines than in normal pancreatic ductal epithelial cells. Data are presented as mean \pm SD $(n=3)$. e Western blot analysis showed that the expression of RFXAP was significantly lower in pancreatic cancercell lines than in normal pancreatic ductal epithelial cells. $\mathbf{f}$ The expression level of RFXAP in pancreatic cancer tissues and normal tissues was detected by immunohistochemistry. Scale bars $50 \mu \mathrm{m}$. g Expression of RFXAP in pancreatic cancer and its association with prognosis. Data are presented as mean \pm SD.

Table 1 Relationship between RFXAP expression and clinicopathological features.

\begin{tabular}{|c|c|c|c|c|}
\hline \multirow[t]{2}{*}{ Parameters } & \multirow[t]{2}{*}{$(n)$} & \multicolumn{2}{|c|}{ RFXAP expressions } & \multirow[t]{2}{*}{$P$ values $(*<0.05)$} \\
\hline & & - & + & \\
\hline Age & & & & 0.683 \\
\hline$<60$ & 18 & 12 & 6 & \\
\hline$\geq 60$ & 28 & 17 & 11 & \\
\hline Gender & & & & 0.936 \\
\hline Male & 22 & 14 & 8 & \\
\hline Female & 24 & 15 & 9 & \\
\hline TNM stage & & & & $0.012^{*}$ \\
\hline$|-| \mid$ & 30 & 15 & 15 & \\
\hline III-IV & 16 & 14 & 2 & \\
\hline Pathological grade & & & & 0.322 \\
\hline Poor & 20 & 11 & 9 & \\
\hline Middle and high & 26 & 18 & 8 & \\
\hline T stage & & & & $0.03^{*}$ \\
\hline $\mathrm{T} 1,2$ & 13 & 5 & 8 & \\
\hline $\mathrm{T} 3,4$ & 33 & 24 & 9 & \\
\hline Lymph node metastasis & & & & 0.322 \\
\hline No & 26 & 18 & 8 & \\
\hline Yes & 20 & 11 & 9 & \\
\hline Distant metastasis & & & & 0.366 \\
\hline Mo & 38 & 23 & 15 & \\
\hline M1 & 8 & 6 & 2 & \\
\hline
\end{tabular}

silencing reduced gamma-H2AX foci levels (Fig. 7d, e), and RFXAP silencing attenuated the growth inhibition effect of fisetin in PDAC cells (Fig. 7f). To further determine the role of KDM4A in pancreatic cancer cells, we depleted KDM4A with CRISPR/Cas9 in PANC-1 cells. Western blot analysis showed the depletion of KDM4A and upregulation of H3K36me3 in PANC-1 cells with or without fisetin treatment (Supplementary Fig. S2A). We also found that depletion of KDM4A enhanced cell viability of PANC-1 cells with fisetin treatment (Supplementary Fig. S2B). Immunofluorescence revealed that depletion of KDM4A enhanced H3K36me3 levels and reduced gamma-H2AX foci levels (Supplementary Fig. S2C, D). Together, these results showed that RFXAP enhanced the DNA damage induced by fisetin via regulation of KDM4A and H3K36me3, thereby impairing DNA HR repair.

Moreover, we found the function of fisetin in enhancing the effect of chemotherapy on pancreatic cancer cells. The results showed that PANC-1 cell viability decreased significantly after co-treatment with $20-\mu \mathrm{M}$ gemcitabine and fisetin (50 or $100 \mu \mathrm{M})$ (Fig. 8a), which showed that fisetin might have a potential to be used as a complementary therapy in cases of chemotherapy resistance by enhancing the chemosensitivity of pancreatic cancer cells.

\section{Discussion}

The low rate of early diagnosis and radical resection of pancreatic cancer remains one of the major challenges faced by clinical oncologists and surgeons ${ }^{1}$. More than $80 \%$ of PDAC patients are at an advanced stage and considered unresectable at the time of diagnosis. Although gemcitabine, S-1, albumin-bound paclitaxel, and other chemotherapy drugs can, to some extent, prolong the survival time of patients with advanced PDAC, after radiochemotherapy, the median survival time of the unresectable patients generally does not exceed 1 year. The efficacies of radiotherapy, targeted therapy, and immunotherapy are also unsatisfactory ${ }^{43-46}$. The nucleotide analogue gemcitabine, which causes cytotoxicity by inducing DNA damage, S-phase arrest, and apoptosis of cancer cells, remains the standard chemotherapy for $\mathrm{PDAC}^{47}$. However, acquired drug resistance to gemcitabine is a major obstacle of chemotherapy for pancreatic cancer $^{48}$. Drug resistance of gemcitabine might be associated with the elevated DNA repair capacity of PDAC $C^{6,7,48}$. Thus, effective targeted therapy or other novel therapies are urgently needed for the treatment of pancreatic cancer.

RFXAP can regulate the transcription of MHC II molecules by binding to CIITA, RFXANK, and RFX5 to form RFX complexes ${ }^{49,50}$. We previously found that, in 


\section{A}

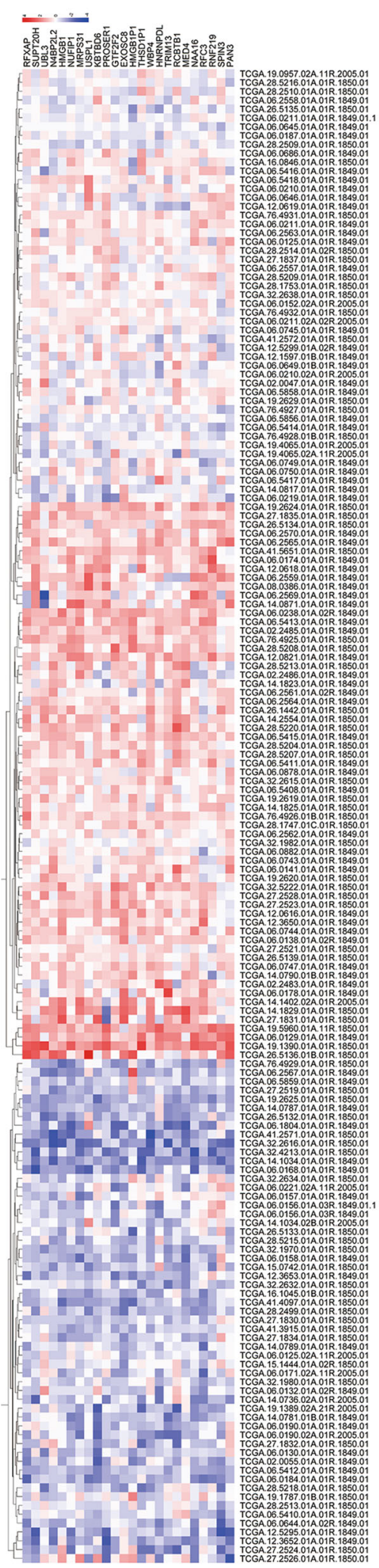

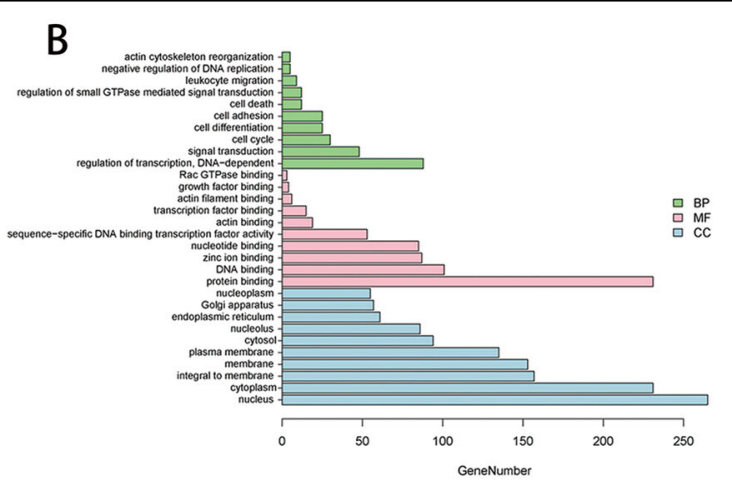

C

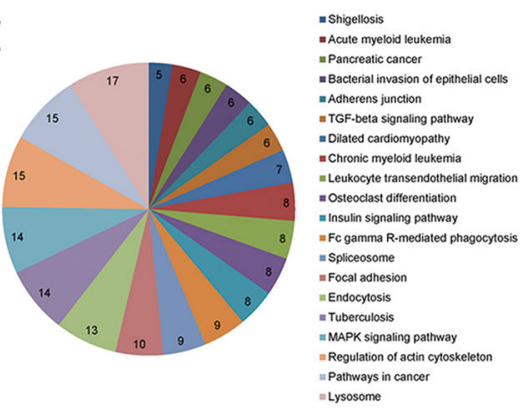

D

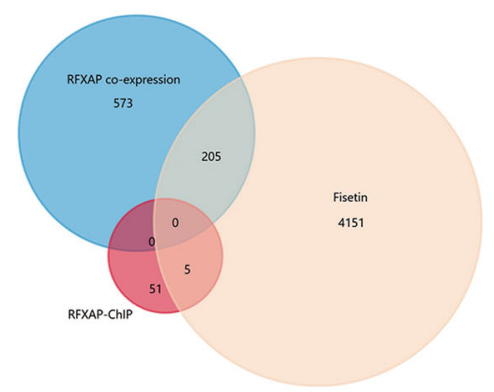

E
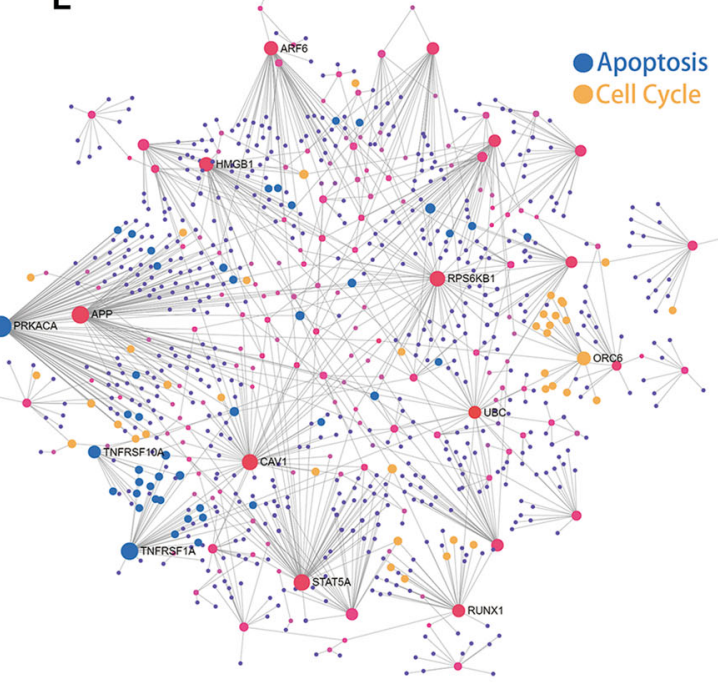

Fig. 4 (See legend on next page.) 
(see figure on previous page)

Fig. 4 Analysis of genes coexpressed with RFXAP, ChIP sequencing, and RNA sequencing in pancreatic cancer. a Heat map of genes with a similarity coefficient of $>0.5$ with RFXAP in The Cancer Genome Atlas pancreatic cancer database. b, c RFXAP expression level was associated with cell-cycle regulation and DNA-damage response. $\mathbf{d}$ Overlapping genes of RFXAP co-expression, ChIP sequencing, and fisetin RNA sequencing were showed by Venn diagrams. e Protein-protein interaction of the overlapping genes of RFXAP co-expression and fisetin RNA-sequencing results was analyzed by NetworkAnalyst. Apoptosis and cell-cycle pathway were enriched, blue means genes related with apoptosis pathway, and yellow means genes related with cell-cycle pathway.

Table. 2 RFXAP interacted with the promoters of 65 genes.

\begin{tabular}{|c|c|c|c|}
\hline RefSeq ID & Symbol & RefSeq ID & Symbol \\
\hline NM_001190452 & MTRNR2L1 & NR_024231 & SNAR-B1 \\
\hline NM_001190470 & MTRNR2L2 & NR_024242 & SNAR-A14 \\
\hline NM_001190702 & MTRNR2L8 & NR_026999 & LINC00265 \\
\hline NM_001286136 & ODF3 & NR_027020 & ANKRD30BL \\
\hline NM_001293091 & CDC27 & NR_027412 & LINC00910 \\
\hline NM_004533 & MYBPC2 & NR_029889 & MIR337 \\
\hline NM_014663 & KDM4A & NR_030386 & MIR663A \\
\hline NM_017761 & PNRC2 & NR_030617 & MIR665 \\
\hline NM_018093 & WDR74 & NR_031608 & MIR663B \\
\hline NM_152837 & SNX16 & NR_033770 & ROCK1P1 \\
\hline NR_003594 & REXO1L2P & NR_037421 & MIR3648-1 \\
\hline NR_024214 & SNAR-A3 & NR_037458 & MIR3687-1 \\
\hline NR_024215 & SNAR-A4 & NR_038368 & LINC00273 \\
\hline NR_024221 & SNAR-C3 & NR_039666 & MIR4461 \\
\hline NR_024223 & SNAR-A5 & NR_039689 & MIR4477B \\
\hline NR_024224 & SNAR-A7 & NR_039705 & MIR4485 \\
\hline NR_024225 & SNAR-A11 & NR_040095 & MIR663AHG \\
\hline NR_024226 & SNAR-A9 & NR_046018 & DDX11L1 \\
\hline NR_024227 & SNAR-A6 & NR_047479 & LINC00854 \\
\hline NR_024228 & SNAR-A8 & NR_051985 & DDX11L9 \\
\hline NR_024229 & SNAR-A10 & NR_103714 & FAM27E2 \\
\hline NR_024230 & SNAR-B2 & NR_103772 & LOC100288162 \\
\hline NR_106735 & MIR6087 & NM_001114091 & $\mathrm{CDC} 27$ \\
\hline NR_106781 & MIR6723 & NM_001256 & CDC27 \\
\hline NR_107045 & MIR8078 & NM_001293089 & CDC27 \\
\hline NR_108107 & FAM230B & NM_001307977 & WDR74 \\
\hline NR_110795 & LOC102723376 & NM_001308208 & ARHGAP39 \\
\hline NR_121645 & RNVU1-8 & NM_022133 & SNX16 \\
\hline NR_121647 & LINC01410 & NM_053280 & ODF3 \\
\hline NR_125957 & LOC101928626 & NM_152836 & ODF3 \\
\hline NR_128711 & MIR3648-2 & NR_027413 & LINC00910 \\
\hline NR_128714 & MIR3687-2 & NR_034090 & DDX11L9 \\
\hline NR_132380 & LINC01596 & & \\
\hline
\end{tabular}

PDAC, miR-212-3p could be transferred into dendritic cells via exosomes to inhibit RFXAP and MHC class II molecules in dendritic cells, thereby inducing immune tolerance $^{10}$. In the current study, RFXAP expression was significantly lower in pancreatic cancer-cell lines and tumor tissues than in the corresponding controls, and negative RFXAP expression was significantly correlated with TNM stage, $\mathrm{T}$ stage, and prognosis. These findings suggest that RFXAP deficiency is a characteristic of PDAC and the expression of RFXAP might be a potential marker of prognosis of PDAC patients.

Fisetin is a natural flavonoid that has been widely studied and inhibits the growth and proliferation of diverse cancer-cell lines. The antitumor effects of fisetin have been indicated to be mediated by altering different signaling pathways in different cellular contexts. Recent studies revealed that fisetin can induce DNA damage and apoptosis in gastric and hepatic cancer ${ }^{25,26}$. Here we found that fisetin induced DNA damage and S-phase arrest, which was similar to the effect of gemcitabine. However, the fisetin molecule differs to the nucleotide analogue gemcitabine. Thus, we hypothesized that fisetin might induce DNA damage through different mechanisms. RNA sequencing found that RFXAP and some genes involved in DNA-damage response, such as RAD51, RAD50, BRCA2, RPA2, and POLD3 were upregulated by fisetin. Bioinformatics analysis showed a correlation between RFXAP and DNA repair genes (i.e., RFXAP expression is positively correlated with $R A D 50$, $B R C A 2$, RPA2, and POLD3 expression) ${ }^{29-41}$. We further explored the role of RFXAP as a transcription factor using ChIP sequencing. We analyzed overlapping genes of RFXAP co-expression, ChIP sequencing, and fisetin RNA sequencing, and found that RFXAP targeted $K D M 4 A$, a specific demethylase for tri- and dimethylated histone H3K36 and H3K9, which can impair DNA HR repair via regulation of $\mathrm{H} 3 \mathrm{~K} 36$ methylation ${ }^{10,51}$. $\mathrm{H} 3 \mathrm{~K} 36 \mathrm{me} 3$ is required for HR repair due to its recruitment effect on lens epithelium-derived growth factor p75 (LEDGF). Following DNA DSBs, LEDGF recruits CtIP to promote resection, and then recruits RPA and RAD51 during HR repair ${ }^{10,11,52,53}$. KDM4A overexpression reduces global H3K36me3 levels, and thereby inhibits RAD51 and RPA foci following DNA damage, which indicates the impairment of HR repair ${ }^{10}$. Then we found that KDM4A was upregulated by fisetin in PDAC, and 
A

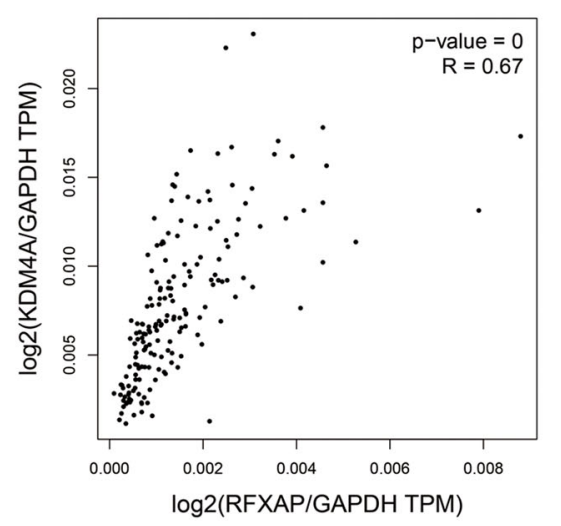

C

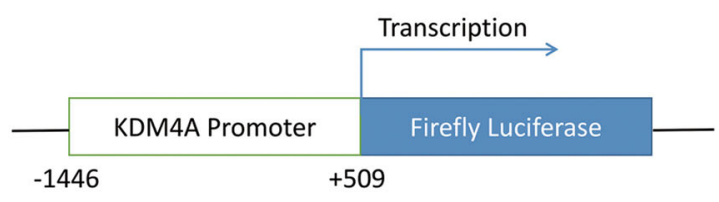

$E$

E
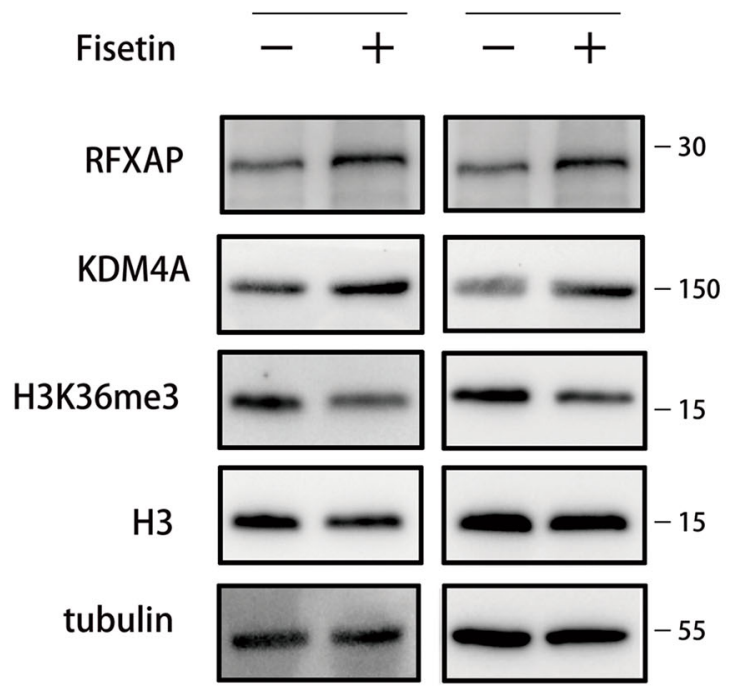

B

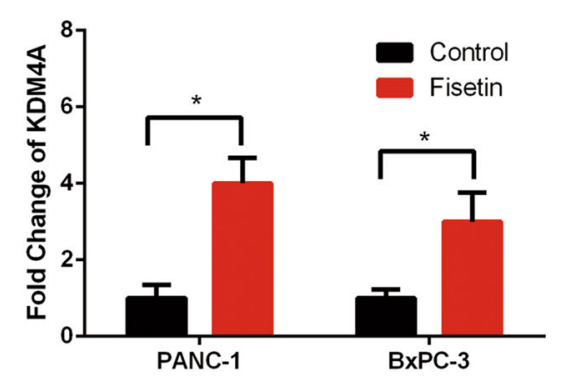

D

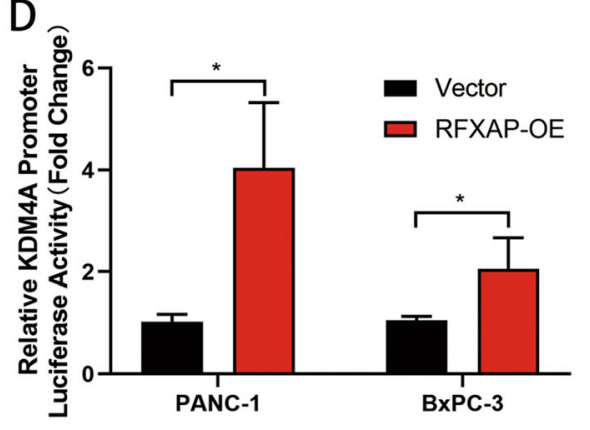

Fig. 5 Expression of KDM4A was upregulated by fisetin. a RFXAP was positively correlated with the expression levels of KDM4A after normalized by GAPDH $\left(R=0.67,{ }^{* * *} P<0.0001, n=89\right)$. $\mathbf{b}$ mRNA expressions of KDM4A in the pancreatic cancer-cell lines PANC-1 and BxPC-3 with treatment of fisetin were detected by qRT-PCR. Data are presented as mean $\pm S D(n=3)$, ${ }^{*} P<0.05$. $\mathbf{c}$, d Transcriptional activity of KDM4A in RFXAP overexpressing and control PDAC cell lines. PANC-1 and BXPC-3 cells were transfected with control vector or RFXAP overexpression vector, then co-transfected with pGL4.23-KDM4A-promoter ( -1446 to +509$)$ and pRL-TK vectors. Luciferase activity was determined $24 \mathrm{~h}$ after transfection. Data are presented as mean \pm SD $(n=3)$. e Expressions of RFXAP, KDM4A, and H3K36me3 in PDAC cells with fisetin treatment were detected by western blot analysis.

RFXAP overexpression could enhance the DNA damage via upregulated KDM4A, thereby attenuating the level of H3K36me3. Moreover, depletion of KDM4A showed lower gamma-H2AX foci levels together with higher H3K36me3 levels and cell viability in PDAC cells with fisetin treatment. We also revealed that RFXAP silencing could downregulate the DNA damage induced by fisetin, via KDM4A-dependent regulation of H3K36 methylation. PDAC is an extremely malignant tumor with genetic instability, which leads to high levels of DNA damage, elevated DNA-damage repair, and serious drug resistance. The deficiency of RFXAP might play an important role in DNA repair and drug resistance. And our results also showed that fisetin might have a potential to be used 
A

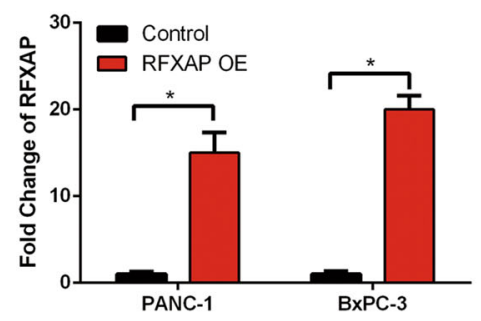

C
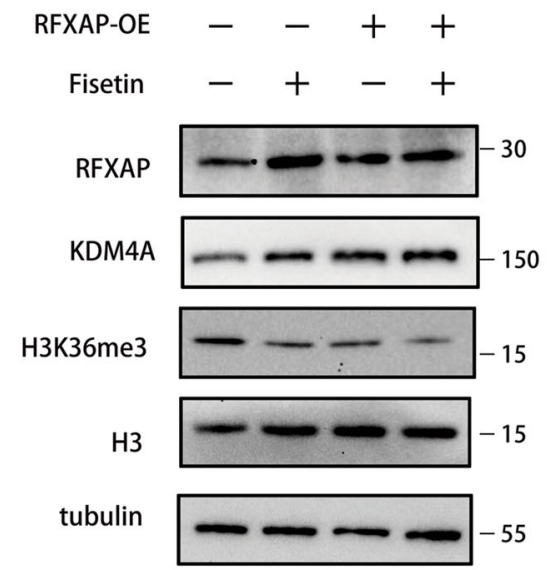

\section{E}
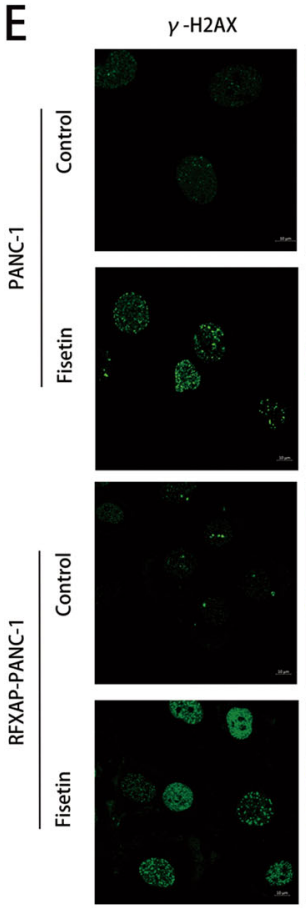

DAPI
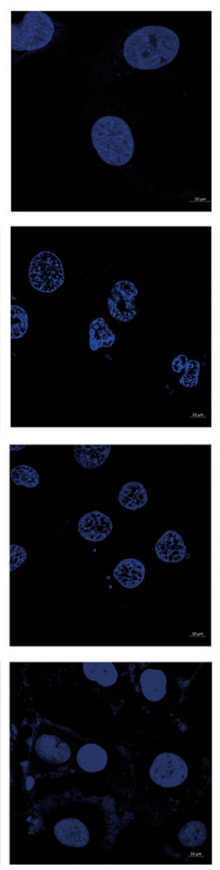

B

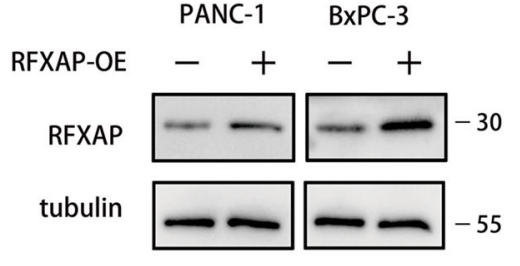

D BxPC-3

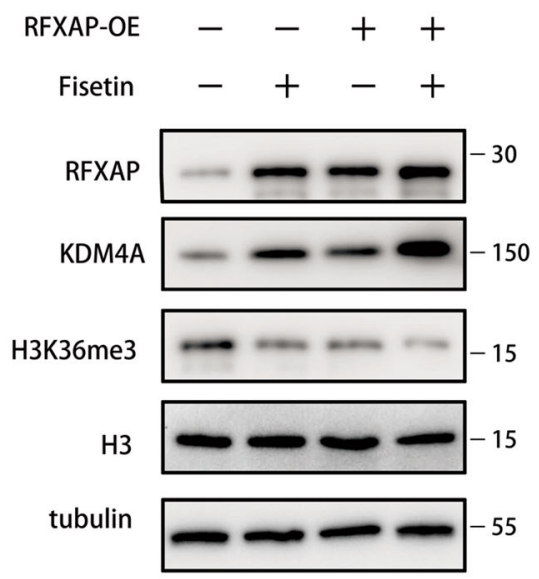

F
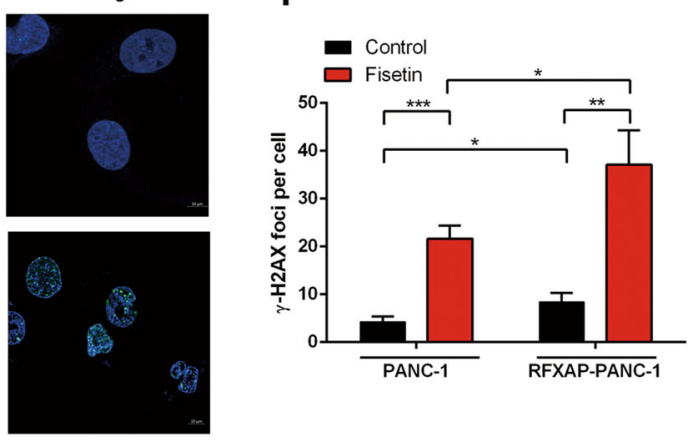

G
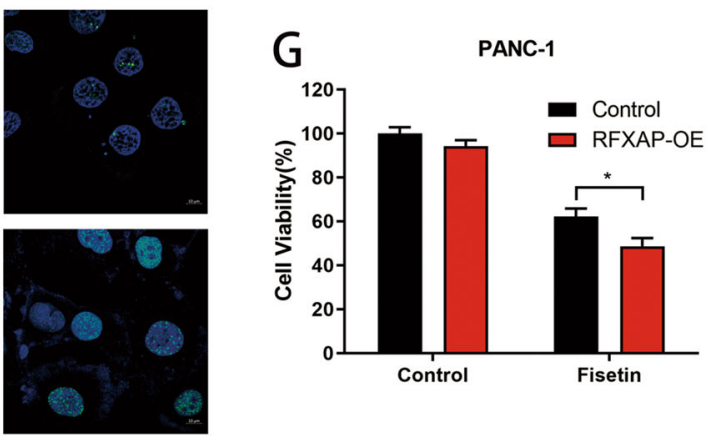

Fig. 6 RFXAP overexpressing enhanced the DNA damage induced by fisetin in pancreatic cancer. a qRT-PCR of RFXAP in control and RFXAP overexpression (RFXAP-OE) PANC-1 and BXPC-3 cells with 100, $50 \mu \mathrm{M}$ fisetin treatment for $48 \mathrm{~h}$. respectively. The results are presented as the mean \pm $\mathrm{SD}(n=3)$, ${ }^{*} P<0.05$. b RFXAP protein expressions were detected by western blot analysis. $\mathbf{c}$, $\mathbf{d}$ Expressions of RFXAP, KDM4A, and H3K36me3 in control and RFXAP overexpression PDAC cells with or without fisetin treatment. e Analyses of DNA damage by observation of the immunofl uorescence of $\mathrm{Y}-\mathrm{H} 2 \mathrm{AX}$ (green) in PDAC cells. RFXAP-PANC-1 cells were treated with fisetin $(100 \mu \mathrm{M})$ for $48 \mathrm{~h}$. The nucleus were counterstained with DAPI (blue). Scale bars $10 \mu \mathrm{m}$. $\mathbf{f}$ Comparative changes in the $\mathrm{\gamma}$-H2AX spots number in control and RFXAP-PANC-1 cells with treatment groups. The spots were counted by manual scoring. Results are mean $\pm S D,{ }^{*} P<0.05$, ${ }^{* *} P<0.01,{ }^{* * *} P<0.001$. g CCK-8 asssy of RFXAP-overexpressing PANC-1 cells. Cells were treated with fisetin (0 or $100 \mu \mathrm{M})$ for $48 \mathrm{~h}$, respectively. The absorbance was measured at $450 \mathrm{~nm}$. Data are presented as mean \pm SD $(n=3)$; ${ }^{*} P<0.05$. 
A

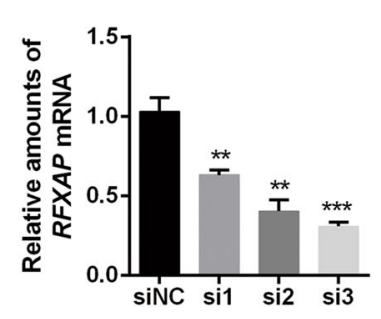

C

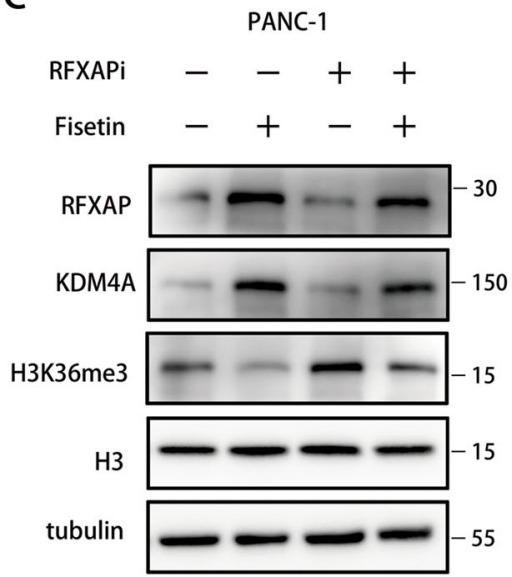

\section{E}
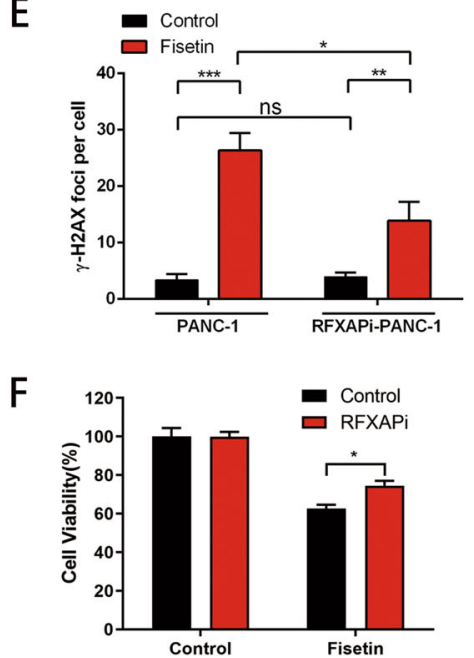

B

PANC-1

RFXAPi siNC si1 si2 si3

RFXAP

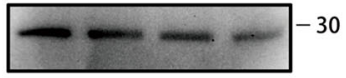

tubulin

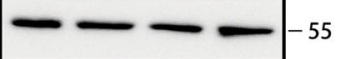

D
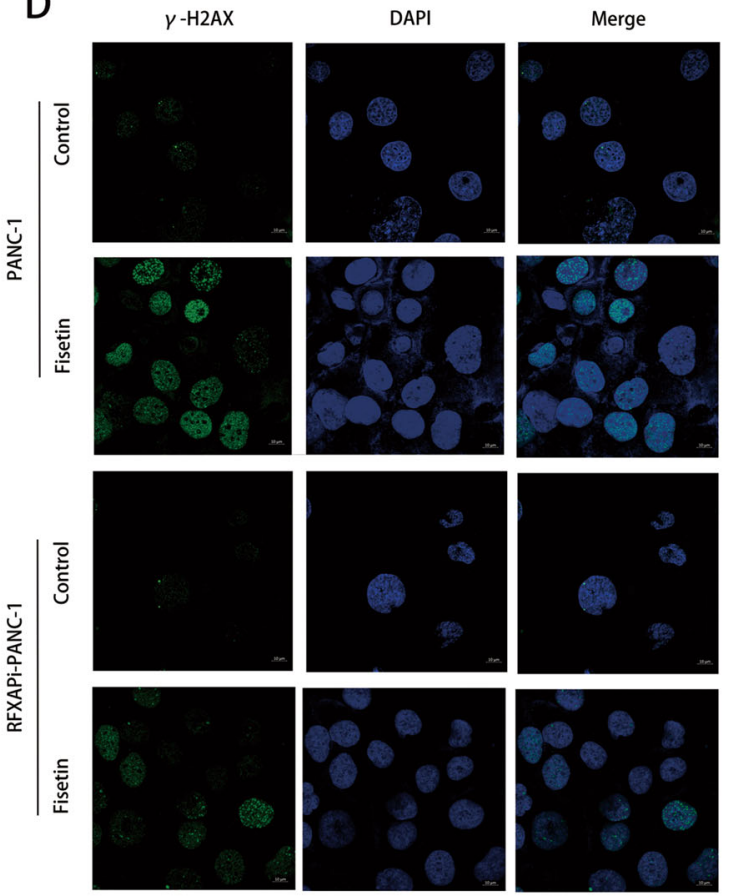

Fig. 7 RFXAP silencing attenuated the DNA damage induced by fisetin in pancreatic cancer. a mRNA levels of RFXAP in control and RFXAP silencing (si1, si2, si3) PANC-1 were detected by qRT-PCR. The results are presented as the mean \pm SD $(n=3)$, ${ }^{* *} P<0.01 .{ }^{* * *} P<0.001$. b RFXAP protein expressions in RFXAP silencing PANC-1 cells were detected by western blot analysis. c Expression levels of KDM4A and H3K36me3 in RFXAP silencing PANC-1 cells, with or without fisetin treatment, were detected by western blot analysis. $\mathbf{d}$ Analyses of DNA damage by observation of the immunofl uorescence of $\mathrm{Y}-\mathrm{H} 2 \mathrm{AX}$ (green) in PDAC cells. RFXAPi-PANC-1 cells were treated with fisetin $(100 \mu \mathrm{M})$ for $48 \mathrm{~h}$. The nucleus were counterstained with DAPI (blue). Scale bars $10 \mu \mathrm{m}$. e Comparative changes in the $\gamma$-H2AX spots number in control and RFXAPi-PANC-1 cells with treatment groups. The spots were counted by manual scoring. Results are mean $\pm S D$, ${ }^{*} P<0.05$, ${ }^{* *} P<0.01$, ${ }^{* *} P<0.001$, \#ns no significance. f CCK-8 asssy of RFXAP silencing PANC-1 cells. Cells were treated with fisetin $(0$ or $100 \mu \mathrm{M})$ for $48 \mathrm{~h}$, respectively. The absorbance was measured at $450 \mathrm{~nm}$. Data are presented as mean $\pm \mathrm{SD}(n=3) ;{ }^{*} P<0.05$.

as a complementary therapy by enhancing the chemosensitivity of pancreatic cancer cells.

In summary, the expression of RFXAP, which was deficient in PDAC, was positively correlated with survival of PDAC patients. Fisetin increased the expression of RFXAP and KDM4A, which impaired HR repair via regulation of histone H3K36 methylation and might attenuate the excessive DNA repair of PDAC, thereby inducing 


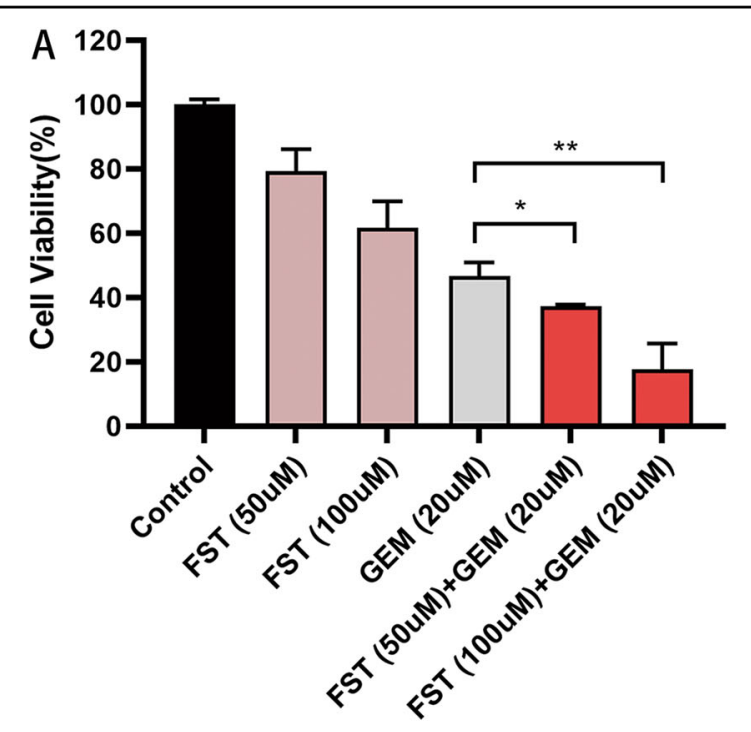

B

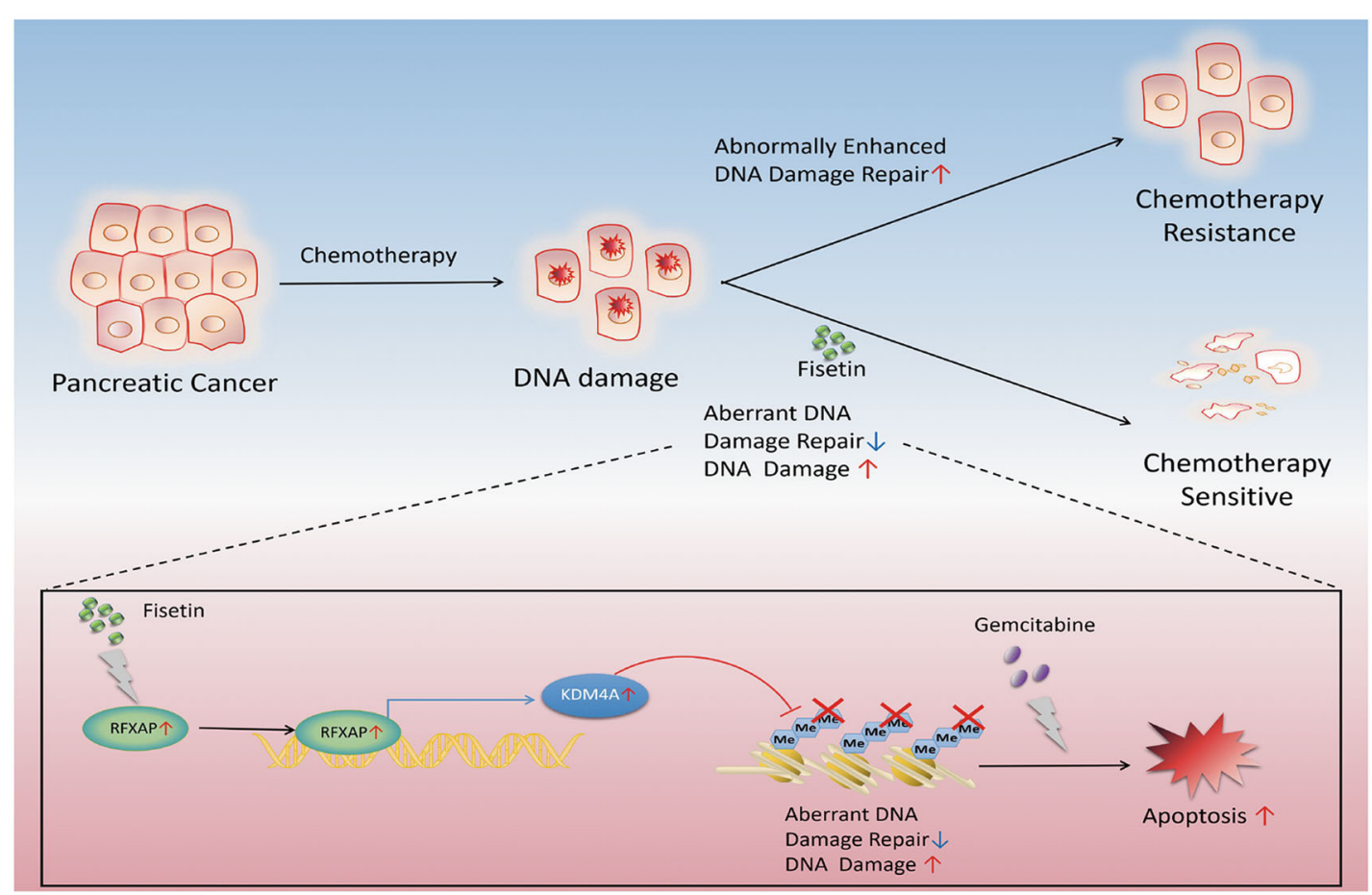

Fig. 8 Fisetin enhances chemosensitivity in pancreatic cancer. a PANC-1 cell viability decreased significantly after co-treatment with 20- $\mu \mathrm{M}$ gemcitabine and fisetin $(50$ or $100 \mu \mathrm{M})$. Data are presented as the mean \pm SD $(n=3)$. b Model indicating that the regulation of DNA damage via RFXAP/KDM4A-dependent histone H3K36 demethylation signaling by fisetin leads to drug sensitivity in pancreatic cancer.

DNA damage and S-phase arrest of PDAC. Thereby fisetin enhances the drug sensitivity of gemcitabine in pancreatic cancer. Together these results indicate that fisetin inhibits cancer-cell proliferation by inducing DNA damage via RFXAP/KDM4A-dependent demethylation of histone H3K36, which might be a novel therapeutic target for PDAC.

\section{Acknowledgements}

This research was supported by the Major Research Project of Science Technology of Zhejiang Province (Grant Number 2019C03048); National Natural Science Foundation of China (Grant Number 81702358); Key Projects of Health and Family Planning Commission of Zhejiang province (Grant Number WKJ-ZJ-1824); Traditional Chinese Medicine Foundation Project of Zhejiang province (Grant Number 2019ZB071); Foundation Project for Medical Science and Technology of Zhejiang province (Grant Number 2019ZH022). 


\section{Author details}

'Department of General Surgery, Sir Run Run Shaw Hospital, School of Medicine, Zhejiang University, Hangzhou 310000, China. 'Department of General Surgery, School of Medicine, Affiliated Hospital of Hangzhou Normal University, Hangzhou 310000, China. ${ }^{3}$ Emergency Department, Sir Run Run Shaw Hospital, School of Medicine, Zhejiang University, Hangzhou 310000 China. ${ }^{4}$ Department of General Surgery, Huzhou Hospital, Zhejiang University School of Medicine, Huzhou 313003 Zhejiang, China. ${ }^{5}$ Innovation Center for Minimally Invasive Technique and Device, Zhejiang University, Hangzhou 310000 Zhejiang, China

\section{Conflict of interest}

The authors declare that they have no conflict of interest.

\section{Ethics approval}

The research protocol was reviewed and approved by the Research Ethics Committee of Sir Run Run Shaw Hospital, School of Medicine, Zhejiang University. All participants or their guardians gave written consent for the use of tissue samples and medical information to be used for scientific research. Animal experiments were approved and performed in accordance with the institutional guidelines for animal care of the animal ethics committee of Zhejiang University.

\section{Publisher's note}

Springer Nature remains neutral with regard to jurisdictional claims in published maps and institutional affiliations.

Supplementary Information accompanies this paper at (https://doi.org/ 10.1038/s41419-020-03019-2).

Received: 24 April 2020 Revised: 5 September 2020 Accepted: 9 September 2020

Published online: 22 October 2020

\section{References}

1. Gillen, S., Schuster, T., Meyer Zum Buschenfelde, C., Friess, H. \& Kleeff, J. Preoperative/neoadjuvant therapy in pancreatic cancer: a systematic review and meta-analysis of response and resection percentages. PLoS Med. 7, e1000267 (2010).

2. Vincent, A., Herman, J., Schulick, R., Hruban, R. H. \& Goggins, M. Pancreatic cancer. Lancet 378, 607-620 (2011).

3. Siegel, R. L., Miller, K. D. \& Jemal, A. Cancer statistics, 2016. CA Cancer J. Clin. 66 7-30 (2016)

4. Chen, W. et al. Cancer statistics in China, 2015. CA Cancer J. Clin. 66, 115-132 (2016).

5. Feitelson, M. A. et al. Sustained proliferation in cancer: mechanisms and novel therapeutic targets. Semin. Cancer Biol. 35, S25-S54 (2015).

6. Nagel, Z. D. et al. DNA repair capacity in multiple pathways predicts chemoresistance in glioblastoma multiforme. Cancer Res. 77, 198-206 (2017).

7. Cheung-Ong, K., Giaever, G. \& Nislow, C. DNA-damaging agents in cancer chemotherapy: serendipity and chemical biology. Chem. Biol. 20, 648-659 (2013).

8. Zhu, J. et al. A novel small molecule RAD51 inactivator overcomes imatinibresistance in chronic myeloid leukaemia. EMBO Mol. Med. 5, 353-365 (2013).

9. Berte, N. et al. Targeting homologous recombination by pharmacological inhibitors enhances the killing response of glioblastoma cells treated with alkylating drugs. Mol. Cancer Ther. 15, 2665-2678 (2016).

10. Pfister, S. X. et al. SETD2-dependent histone H3K36 trimethylation is required for homologous recombination repair and genome stability. Cell Rep. 7 2006-2018 (2014)

11. Daugaard, M. et al. LEDGF (p75) promotes DNA-end resection and homologous recombination. Nat. Struct. Mol. Biol. 19, 803-810 (2012).

12. Aymard, F. et al. Transcriptionally active chromatin recruits homologous recombination at DNA double-strand breaks. Nat. Struct. Mol. Biol. 21, 366-374 (2014).

13. Gokturk, B., Artac, H., van Eggermond, M. J., van den Elsen, P. \& Reisli, I. Type III bare lymphocyte syndrome associated with a novel RFXAP mutation: a case report. Int J. Immunogenet. 39, 362-364 (2012).
14. Hanna, S. \& Etzioni, A. MHC class I and II deficiencies. J. Allergy Clin. Immunol. 134, 269-275 (2014).

15. Ding, G. et al. Pancreatic cancer-derived exosomes transfer miRNAs to dendritic cells and inhibit RFXAP expression via miR-212-3p. Oncotarget 6 29877-29888 (2015)

16. $\mathrm{Ma}$, C. et al. miR-212 promotes pancreatic cancer cell growth and invasion by targeting the hedgehog signaling pathway receptor patched-1. J. Exp. Clin. Cancer Res. 33, 54 (2014).

17. Park, J. K. et al. miR-132 and miR-212 are increased in pancreatic cancer and target the retinoblastoma tumor suppressor. Biochem. Biophys. Res. Commun. 406, 518-523 (2011)

18. Wu, Z., Zhou, L., Ding, G. \& Cao, L. Overexpressions of miR-212 are associated with poor prognosis of patients with pancreatic ductal adenocarcinoma. Cancer Biomark. 18, 35-39 (2017)

19. Surmann, E. M et al. Association of high CD4-positive T cell infiltration with mutations in HLA class II-regulatory genes in microsatellite-unstable colorectal cancer. Cancer Immunol. Immunother. 64, 357-366 (2015).

20. Arai, Y. et al. Dietary intakes of flavonols, flavones and isoflavones by Japanese women and the inverse correlation between quercetin intake and plasma LDL cholesterol concentration. J. Nutr. 130, 2243-2250 (2000).

21. Hanneken, A., Lin, F. F., Johnson, J. \& Maher, P. Flavonoids protect human retinal pigment epithelial cells from oxidative-stress-induced death. Invest. Ophthalmol. Vis. Sci. 47, 3164-3177 (2006)

22. Higa, S. et al. Fisetin, a flavonol, inhibits TH2-type cytokine production by activated human basophils. J. Allergy Clin. Immunol. 111, 1299-1306 (2003).

23. Khan, N., Afaq, F., Syed, D. N. \& Mukhtar, H. Fisetin, a novel dietary flavonoid causes apoptosis and cell cycle arrest in human prostate cancer LNCaP cells. Carcinogenesis 29, 1049-1056 (2008).

24. Kim, J. A. et al. Fisetin, a dietary flavonoid, induces apoptosis of cancer cells by inhibiting HSF1 activity through blocking its binding to the hsp70 promoter. Carcinogenesis 36, 696-706 (2015).

25. Sabarwal, A., Agarwal, R. \& Singh, R. P. Fisetin inhibits cellular proliferation and induces mitochondria-dependent apoptosis in human gastric cancer cells. Mol. Carcinog. 56, 499-514 (2017).

26. Kim, J. Y., Jeon, Y. K., Jeon, W. \& Nam, M. J. Fisetin induces apoptosis in Huh-7 cells via downregulation of $\mathrm{BIRC} 8$ and $\mathrm{BCl} 2 \mathrm{~L} 2$. Food Chem. Toxicol. 48 , 2259-2264 (2010).

27. Hytti, M. et al. Two dietary polyphenols, fisetin and luteolin, reduce inflammation but augment DNA damage-induced toxicity in human RPE cells. J. Nutr. Biochem 42, 37-42 (2017).

28. Tang, Z. et al. GEPIA: a web server for cancer and normal gene expression profiling and interactive analyses. Nucleic Acids Res. 45, W98-W102 (2017).

29. Al Abo, M. et al. TDP1 is critical for the repair of DNA breaks induced by sapacitabine, a nucleoside also targeting ATM- and BRCA-deficient tumors. Mol. Cancer Ther. 16, 2543-2551 (2017).

30. Cassidy, L. D., Liau, S. S. \& Venkitaraman, A. R. Chromosome instability and carcinogenesis: insights from murine models of human pancreatic cancer associated with BRCA2 inactivation. Mol. Oncol. 8, 161-168 (2014).

31. Fan, C. et al. RAD50 germline mutations are associated with poor survival in BRCA1/2-negative breast cancer patients. Int J. Cancer 143, 1935-1942 (2018).

32. Anand, R., Ranjha, L., Cannavo, E. \& Cejka, P. Phosphorylated CtIP functions as a co-factor of the MRE11-RAD50-NBS1 endonuclease in DNA end resection. Mol. Cell 64, 940-950 (2016).

33. Baude, A. et al. Hepatoma-derived growth factor-related protein 2 promotes DNA repair by homologous recombination. Nucleic Acids Res. 44, 2214-2226 (2016).

34. Syed, A. \& Tainer, J. A. The MRE11-RAD50-NBS1 complex conducts the orchestration of damage signaling and outcomes to stress in DNA replication and repair. Annu. Rev. Biochem. 87, 263-294 (2018).

35. Ahn, J. W. et al. SERBP1 affects homologous recombination-mediated DNA repair by regulation of CtIP translation during S phase. Nucleic Acids Res. $\mathbf{4 3}$ 6321-6333 (2015)

36. Shi, W. et al. The role of RPA2 phosphorylation in homologous recombination in response to replication arrest. Carcinogenesis 31, 994-1002 (2010).

37. Liu, S et al. Inhibition of ATR potentiates the cytotoxic effect of gemcitabine on pancreatic cancer cells through enhancement of DNA damage and abrogation of ribonucleotide reductase induction by gemcitabine. Oncol. Rep. 37, 3377-3386 (2017)

38. Zhou, Z. et al. Pold3 is required for genomic stability and telomere integrity in embryonic stem cells and meiosis. Nucleic Acids Res. 46, 3468-3486 (2018). 
39. Miquel, $C$. et al. Frequent alteration of DNA damage signalling and repair pathways in human colorectal cancers with microsatellite instability. Oncogene 26, 5919-5926 (2007).

40. Buis, J. et al. Mre11 nuclease activity has essential roles in DNA repair and genomic stability distinct from ATM activation. Cell 135, 85-96 (2008).

41. Venkitaraman, A. R. Cancer suppression by the chromosome custodians, BRCA1 and BRCA2. Science 343, 1470-1475 (2014).

42. Xia, J., Gill, E. E. \& Hancock, R. E. NetworkAnalyst for statistical, visual and network-based meta-analysis of gene expression data. Nat. Protoc. 10 823-844 (2015).

43. Gupta, R., Amanam, I. \& Chung, V. Current and future therapies for advanced pancreatic cancer. J. Surg. Oncol. 116, 25-34 (2017).

44. Ilic, M. \& Ilic, I. Epidemiology of pancreatic cancer. World J. Gastroenterol. 22, 9694-9705 (2016).

45. Jones, W. E. 3rd et al. ACR appropriateness criteria(R) resectable pancreatic cancer. Am. J. Clin. Oncol. 40, 109-117 (2017).

46. Kuroczycki-Saniutycz, S. et al. Prevention of pancreatic cancer. Contemp. Oncol. (Pozn.) 21, 30-34 (2017).
47. Kamisawa, T., Wood, L. D., Itoi, T. \& Takaori, K. Pancreatic cancer. Lancet $\mathbf{3 8 8}$ 73-85 (2016).

48. O'Reilly, E. M. \& Abou-Alfa, G. K. Cytotoxic therapy for advanced pancreatic adenocarcinoma. Semin. Oncol. 34, 347-353 (2007).

49. Bushway, M., Cycon, K. A., Mulvaney, K. \& Murphy, S. P. Coordinate loss of MHC class $\|$ expression in the diffuse large B cell lymphoma cell line OCl-Ly2 is due to a novel mutation in RFX-AP. Immunogenetics 62, 109-116 (2010).

50. Peretti, M., Villard, J., Barras, E., Zufferey, M. \& Reith, W. Expression of the three human major histocompatibility complex class II isotypes exhibits a differential dependence on the transcription factor RFXAP. Mol. Cell. Biol. 21, 5699-5709 (2001).

51. Mallette, F. A. et al. RNF8- and RNF168-dependent degradation of KDM4A JMJD2A triggers 53BP1 recruitment to DNA damage sites. EMBO J. 31, 1865-1878 (2012).

52. Eidahl, J. O. et al. Structural basis for high-affinity binding of LEDGF PWWP to mononucleosomes. Nucleic Acids Res. 41, 3924-3936 (2013).

53. Sartori, A. A. et al. Human CtIP promotes DNA end resection. Nature 450, 509-514 (2007). 